\title{
Conservación y mantenimiento del patrimonio inmueble: la Cruz de Término de El Masnou
}

\author{
Manuel Ángel Iglesias Campos; M. Núria Avecilla Palau y Marta Llach Berné
}

Resumen: La conservación y el mantenimiento del patrimonio inmueble tiene un coste económico que debe ajustarse a los presupuestos de las instituciones responsables de su tutela, y aún más en la actualidad. Adaptar los estudios a las necesidades reales de la intervención y obtener información suficiente para el seguimiento posterior es de vital importancia y permite actuar según los criterios establecidos. En este trabajo se expone un ejemplo de colaboración entre instituciones en el que se adecuaron los medios técnicos y los recursos disponibles para plantear la intervención y establecer un protocolo detallado de seguimiento. Sistematizando las necesidades reales, a partir de los primeros análisis visuales, se realizó un estudio previo sencillo que facilitó información esencial para efectuar los trabajos y proponer unos controles periódicos. Tras más de un año del inicio del proyecto, la racionalización de los objetivos y la colaboración y el compromiso entre las instituciones y las personas implicadas, han permitido alcanzar los propósitos programados.

Palabras clave: Cruz de término, arenisca silícea, costra negra, alveolización, biodeterioro, programa de mantenimiento.

\section{Conservation and maintenance of built heritage: The wayside Cross in El Masnou}

Abstract: The cost of preservation and maintenance of built heritage must fit the budgets of the institutions responsible for their care and upkeep, especially at present. Adapting preliminary studies to the actual requirements of intervention and obtaining enough information for its continued monitoring is extremely important, and allows an intervention according to the established criteria. This paper presents an example of collaborative action between institutions. Technical means and resources were fitted to the intervention and used to set up a detailed monitoring protocol. Organizing actual needs from the initial visual analysis, a simple previous study was carried out to provide essential information dealing with the work schedule and subsequent monitoring. After more than a year into the project, rationalization of specific requirements, collaboration and commitment between institutions and people involved has allowed the achievement of the outlined objectives.

Key words: Cross, siliceous sandstone, black crust, alveolization, biodeterioration, maintenance program.

\section{Conservação e manutenção de patrimonio imóvel: o cruzeiro de El Masnou.}

Resumo: A conservação e a manutenção de patrimonio imóvel tem um custo económico que deve ajustar-se aos orçamentos das instituições responsáveis pela sua tutela e, ainda mais na actualidade. Adaptar os estudos às necessidades reais da intervenção e obter informação suficiente para dar seguimento posterior, é de vital importancia e permite actuar segundo os criterios estabelecidos. Neste trabalho, expõe-se um exemplo de colaboração entre instituições em que se adequaram os meios técnicos e os recursos disponíveis para planear a intervenção e estabelecer um protocolo detalhado de acompanhamento. Sistematizando as necessidades reais, a partir das primeiras análises visuais, realizou-se um estudo prévio simples que providenciou informação essencial para efectuar os trabalhos e propor alguns controles periódicos. Após mais de um ano sobre o início do projecto, a racionalização dos objectivos, a colaboração e o compromisso entre as instituições e as pessoas implicadas, permitiram alcançar os objectivos traçados.

Palavras-chave: Cruzeiro, arenito silicioso, crosta negra, alveolização, biodeterioração, programa de manutenção. 


\section{Introducción}

Los estudios y trabajos realizados en la Cruz de Término de El Masnou se enmarcan en el programa de soporte a la conservación y restauración que la Xarxa de Museus Locals que la Oficina de Patrimonio Cultural (OPC) de la Diputación de Barcelona viene desarrollando desde 2001. Una de las líneas del Programa de Conservación y Restauración pretende implementar planes de conservación preventiva mediante inspecciones periódicas - y de seguimiento a medio y largo plazo - que permitan mantener el Patrimonio Cultural Local en las mejores condiciones posibles, aunque en algunos casos, dependiendo de la obra y de las necesidades del municipio, supone también intervenir in situ con personal del Laboratorio de Conservación y Restauración de la OPC. Gracias a esta metodología de trabajo, adaptada a los medios técnicos y económicos disponibles, y siguiendo los criterios establecidos para estas intervenciones (AA.VV 2013, AA.VV 2003), se pretende llevar un seguimiento documentado de estos bienes culturales y estudiar la evolución y la eficacia de los tratamientos realizados.

Partiendo de los objetivos del Programa, esta intervención se realizó a petición del Museo de La Náutica de El Masnou. La finalidad primera era determinar el estado de conservación de la Cruz de Término y posteriormente, a partir de unos estudios previos, proponer y realizar los tratamientos necesarios y elaborar una propuesta de seguimiento y control.

A pesar del mal estado de conservación, principalmente del capitel, la voluntad del consistorio por mantener la
Cruz in situ fundamentaba un proyecto y una intervención encaminados a frenar los procesos de degradación e implementar, a continuación, un plan periódico de conservación. El programa de control y mantenimiento serviría, además, para evaluar la intervención realizada, para determinar si los procesos de degradación continuaban y a qué ritmo, y para proponer soluciones que permitiesen la conservación en su ubicación actual.

\section{La Cruz de Término}

La Cruz de Término de El Masnou es uno de los elementos patrimoniales inmuebles con mayor valor histórico-artístico del municipio y el único testimonio medieval que se conserva. Es una obra devocional de estilo gótico tardío, similar estilísticamente a las cruces de término de las poblaciones vecinas de Teià y Alella.

El Masnou es un municipio situado a $17 \mathrm{~km}$ al noreste de Barcelona, en la comarca del Maresme. Orográficamente, se encuentra entre la Sierra Litoral y el mar. Tiene un clima mediterráneo suave, con veranos calurosos y húmedos e inviernos templados. Las lluvias, irregulares y escasas, se concentran en otoño y primavera, y ocasionalmente pueden ser muy intensas. Aunque no es una población industrial, su cercanía con el área metropolitana de Barcelona hace que se encuentre en una zona de contaminación importante (Actium Patrimoni Cultural, SL. 2010, pp. 6-17).

Antiguamente, cuando El Masnou aún no existía como municipio y sin que se sepa con exactitud, la Cruz de Término se encontraba en la actual avenida Joan XXIII, delimi-
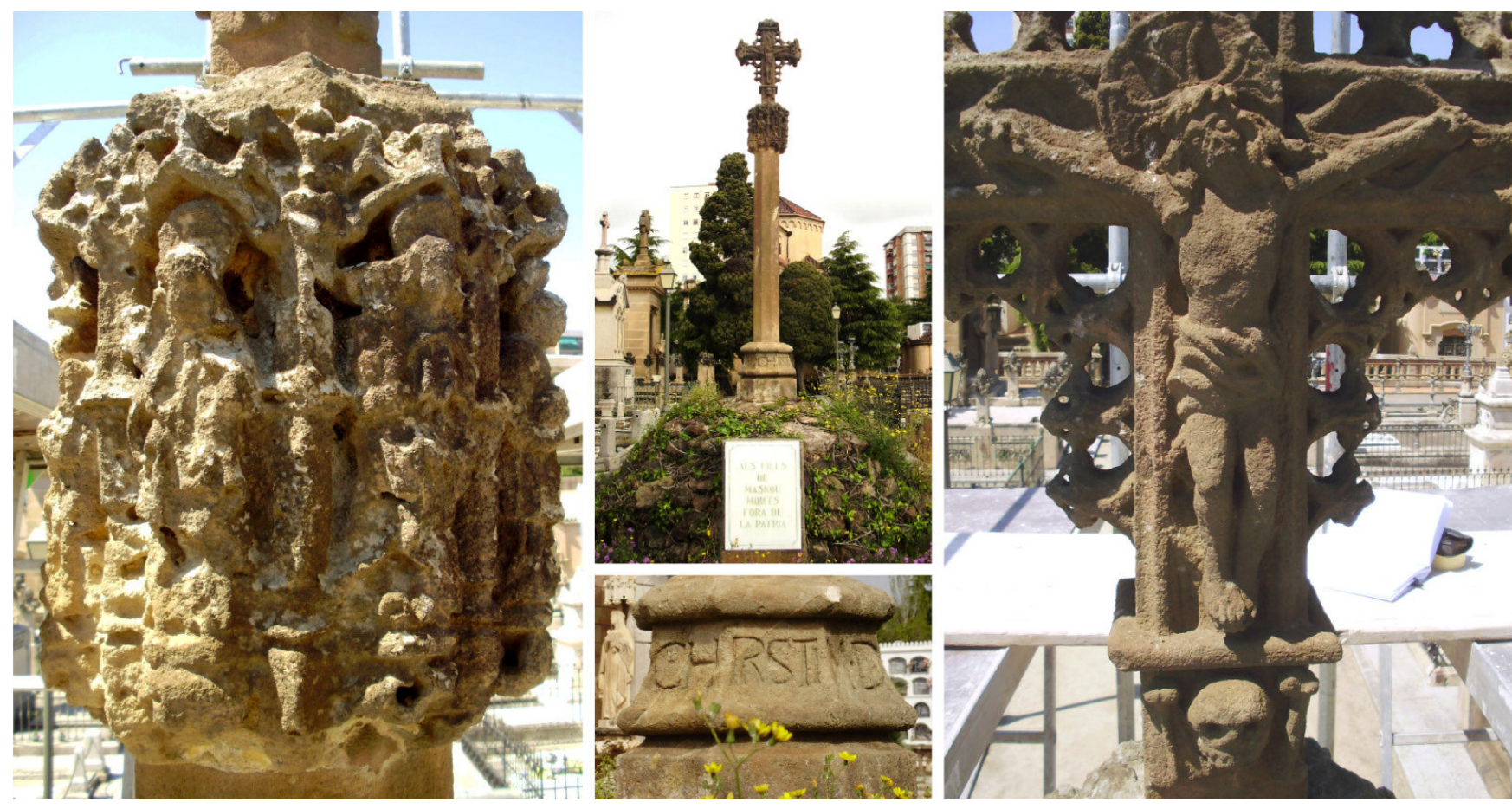

Figura 1. Cruz de Término y detalles de las piezas con decoración escultórica y epigrafía. @ Manuel A. Iglesias Campos. 

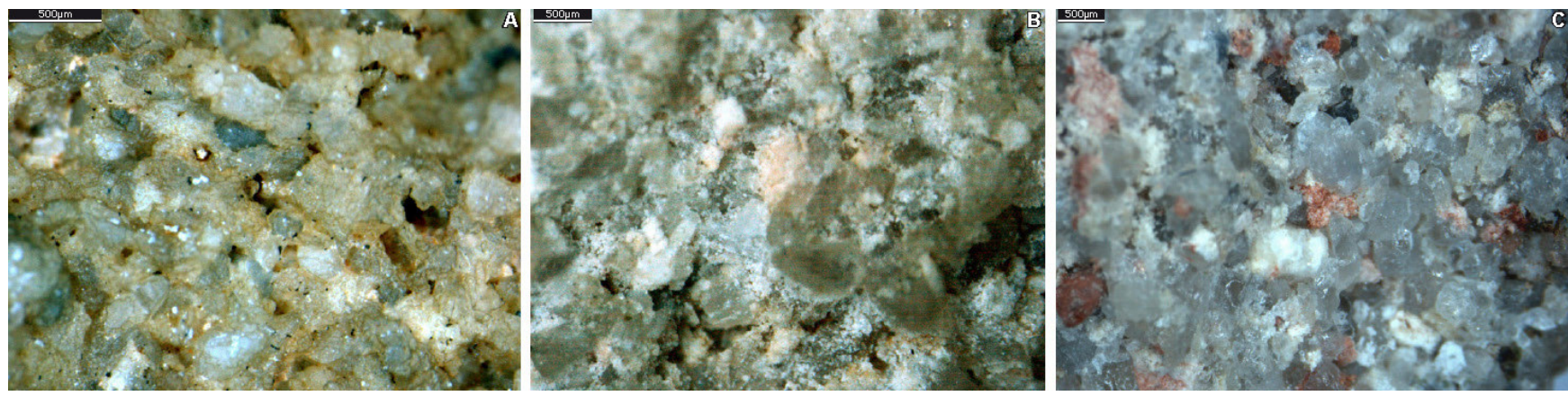

Figura 2. Material pétreo: $(A)$ anverso de una muestra, $(B, C)$ reverso de dos de las muestras. @ Manuel A. Iglesias Campos.

tando los términos de Teià y Alella. Hacia 1818, cuando en la necrópolis de la iglesia de Sant Pere se construyó el conocido como Cementerio Viejo, la Cruz se trasladó a este nuevo emplazamiento. En 1868, al construirse el actual cementerio, proyectado por el arquitecto Miquel Garriga i Roca en la parte alta del municipio, se volvió a trasladar, reubicándose, sin conocer la fecha exacta, en el centro del nuevo camposanto sobre un basamento de ladrillo recubierto con mampostería irregular y rodeado de un parterre quadrilobular ${ }^{1}$. Durante el siglo $X X$, se colocaron sobre este basamento tres placas conmemorativas que recogen hechos históricos de la población. El cementerio, actualmente en proceso de recuperación, es para el municipio un conjunto artístico de gran valor testimonial.

La Cruz, declarada BCIN en $1963^{2}$, está esculpida en piedra y conserva diferentes morteros de unión y recubrimientos cromáticos artificiales ${ }^{3}$. Consta de basa, fuste, capitel y cruz propiamente dicha ${ }^{4}$, y mide aproximadamente $3 \mathrm{~m}$ de altura sin contar el basamento actual ${ }^{5}$. La basa es octogonal y moldurada, con la inscripción ANNO CHRISTI $M D$. El fuste, también octogonal, se remata con un capitel con la imagen de ocho santos dentro de hornacinas - no identificados por su mal estado de conservación-. En la cruz se representa a Cristo crucificado con la calavera a los pies en una de sus caras; en la otra, a la Virgen con el niño en brazos; y en los laterales, motivos vegetales y florones calados [Figura 1].

Se desconoce si la disposición y orientación actuales se corresponden con las iniciales y si los morteros y recubrimientos cromáticos podrían ser originales ${ }^{6}$. Tampoco se sabe si tras las reubicaciones se realizaron tratamientos de limpieza y/o protección, aunque la observación directa parece indicar que sí.

\section{Estudios previos}

A partir de los primeros análisis visuales se decidió realizar un estudio previo sencillo, con un coste asumible dentro del presupuesto del proyecto, que facilitase la información suficiente para la intervención y para programar los controles posteriores. Así, tras una observación in situ mediante microscopio digital portátil y macrofoto- grafía, se tomaron muestras directas con las que estudiar en laboratorio los diferentes materiales (pétreo, morteros y recubrimientos cromáticos artificiales) y los indicadores de alteración (costras, biodeterioro y sales solubles). Las muestras se seleccionaron por su representatividad aprovechando pequeños desprendimientos en fisuras y descamaciones. Los equipos empleados en el laboratorio fueron el microscopio estereoscópico Leica M165C con 7,3-120x y software de visualización y medición Leica Stereo Explorer 3D, el conductímetro Crison EC-Meter Basic30+, y el pH-metro Crison pH-Meter basic 20+.

La información se recogió en fotografías generales y de detalle que se utilizaron, además, como soporte directo para la cartografía. También se confeccionaron diferentes tablas en las que sintetizar las observaciones y los resultados del todo el proceso.

\section{Material pétreo}

El material escultórico es una arenisca silícea con ligeras variaciones según la pieza concreta [Figura 2]. Su textura es detrítica y granosoportada, predominando granos equigranulares con formas subangulosas, que presentan contactos completos y entre los que existe porosidad intergranular. De manera general los granos esqueléticos corresponden a cuarzo y a porcentajes variables de fragmentos de roca, feldespatos, y, más accesoriamente, moscovita. El material intersticial que aparece entre los granos se caracteriza por una notable variabilidad ya que se identifica desde una matriz arcillosa a una cementación que puede variar de silícea a carbonatada. Petrográficamente se puede clasificar como una arenisca lítica y sus características se corresponden con la arenisca de Montjuïc (Esbert et al. 1999, Farrando Boix 1999, GómezGras et al. 2001).

La textura de acabado superficial es lisa, o más bien microrugosa por el tamaño de los granos minerales -entre $150-300 \mu \mathrm{m}$ - El análisis de la rugosidad superficial en muestras poco alteradas indica una diferencia media entre picos y valles entre $100-200 \mu \mathrm{m}$.

En el fuste y en la basa se distingue una capa superfi- 
cial de aspecto no pétreo y ligeramente cristalina. La observación del anverso de las muestras con microscopio estereoscópico confirmó su presencia. En el reverso, en cambio, se apreciaba cierta descohesión entre los granos minerales por pérdida de las fases de unión y un relativo deterioro de las formas de los granos [Figura 2]. El análisis por absorción de gota de agua en todas las muestras de material pétreo, observado con microscopio estereoscópico, permitió comprobar, además, que en el anverso no se producía casi absorción pero sí en el reverso, lo que podría relacionarse con la presencia de algún tratamiento que reducía la penetración del agua desde el exterior.

\section{Morteros}

En las uniones entre las piezas se conservan diferentes morteros que podrían estar relacionados con los diversos traslados y montajes de la Cruz. Son morteros de cal aérea y/o morteros hidráulicos de unión y/o de rejuntado localizados entre la cruz, el capitel y el fuste —el encaje entre el fuste y la basa está hecho con plomo- [Figura 3]. Puntualmente se observan también morteros de cemento en el basamento de mampostería.

Los morteros mayoritarios son de coloración rosácea y por sus características, su ubicación y su disposición frente al resto de los conservados, podrían corresponder al montaje de finales del XIX. Entre capitel y fuste tienen una forma rectangular, recubren cuñas de madera -en la cara $\mathrm{N}$, donde se había perdido parte de este mortero, se observan restos de estas cuñas-y sobre ellos se aprecia un recubrimiento cromático artificial de tonali- dad rosácea. Tienen una textura y composición similar a la de morteros hidráulicos documentados en el mismo periodo en esta región (Iglesias Campos et al. 2014), con áridos silíceos de tamaño arena fina y gruesa, morfología principalmente angulosa y subredondeada, y dosificación aproximada de 1:4 [Figura 3M1].

Dos tipologías minoritarias se conservan también en la unión entre capitel y fuste. Una se corresponde a la de un mortero de cal con áridos silíceos de tamaño arena media y fina con morfología principalmente angulosa y subredondeada y dosificación aproximada de 1:3. Tiene, además, un recubrimiento cromático artificial de color amarillento. Este mortero solo se ha localizado en tres de las caras de unión entre capitel y fuste, está colocado directamente sobre la piedra [Figura 3M2] y en algunas zonas aparece por debajo del rosáceo. La otra tipología se corresponde a la de un mortero de cal sin apenas árido. Por su ubicación debió de utilizarse para unir el capitel y el fuste en algún momento de su historia y, cuando aparece, está por debajo del resto de los morteros [Figura 3M3].

Es necesario indicar que en análisis microquímicos de estos morteros con ácido clorhídrico se observó una fuerte efervescencia en el reverso de la muestra, pero no en el anverso. Al igual que lo comentado al hablar de la piedra, los análisis por absorción de gota de agua de las muestras confirmaron que en el anverso no se producía absorción y sí, en cambio, en el reverso. Esto parecía indicar de nuevo la existencia de un tratamiento en la parte exterior que reducía la penetración del agua y, en este caso también, la del ácido.
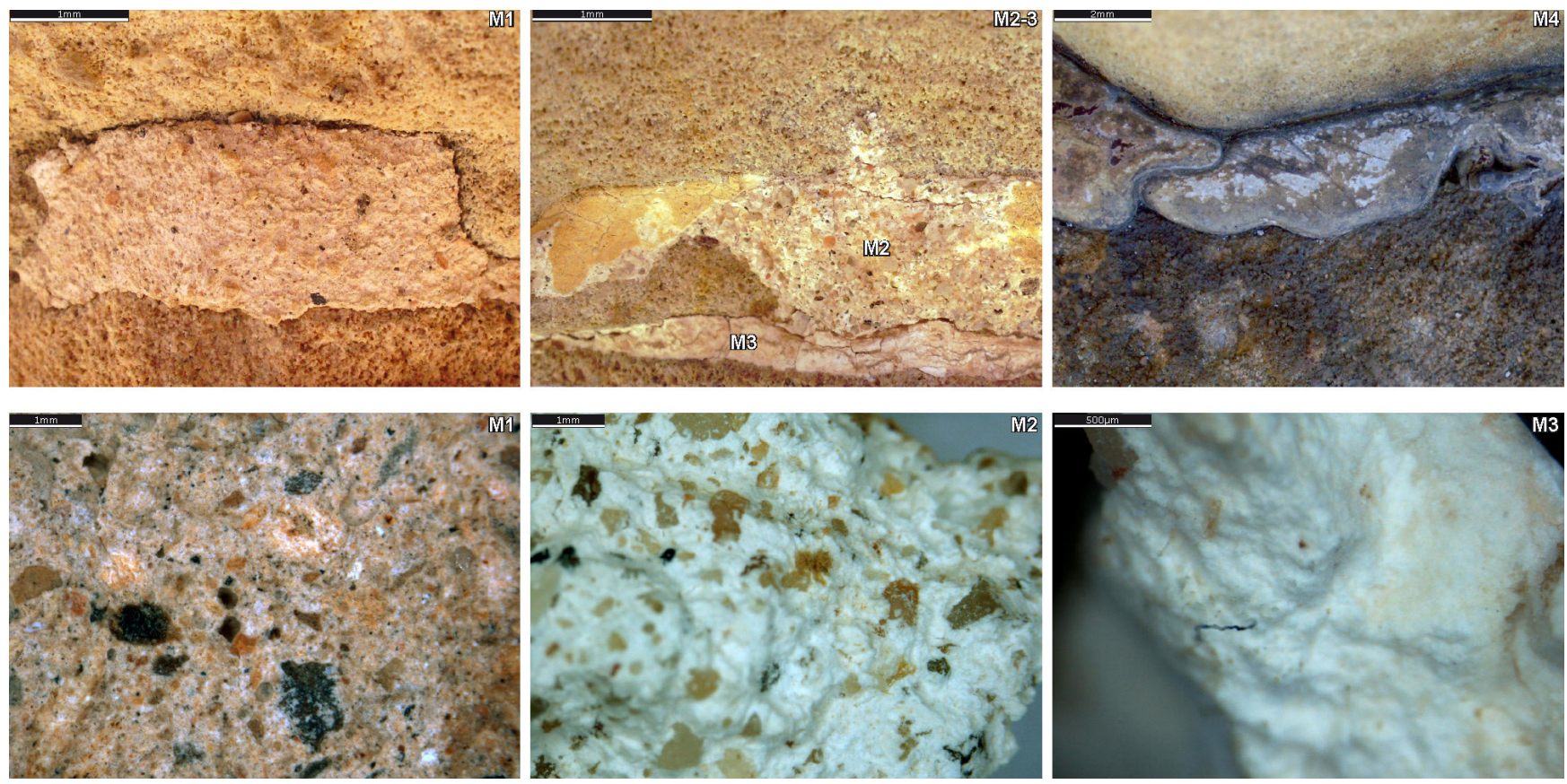

Figura 3. Sistemas de unión entre las partes de la Cruz: macrofotografías (superior) e imágenes a microscopio estereoscópico (inferior) -(M1) mortero de unión capitel-fuste, (M2) mortero de recubrimiento en junta entre capitel-fuste con cromatización ocre, (M3) mortero de unión capitel-fuste, (M4) encaje con plomo entre fuste y basa- . ๑ Manuel A. Iglesias Campos. 
Existen otros morteros que, por su carácter puntual y por no estar relacionados tipológicamente con ninguno de los utilizados en los montajes de la Cruz, aquí no se consideran. Este sería el caso del empleado en dos pequeñas reposiciones del fuste - mortero de cal con árido silíceo grueso- y de los de rejuntado del basamento de mampostería - morteros de cemento con diferentes composiciones y proporciones que parecen relacionarse con intervenciones periódicas de mantenimiento-.

\section{Recubrimientos cromáticos artificiales}

Como se ha apuntado, sobre estos morteros se conservan dos recubrimientos cromáticos artificiales - uno de tonalidad rosácea y el otro de tonalidad amarillenta- que por su disposición apuntan a momentos diferentes [Figura 4]. El de tonalidad rosácea [Figura 4A] tiene un aspecto similar al de una lechada de mortero pigmentada, sin conocer exactamente su composición. Rellena los espacios intergranulares y los valles de la textura de acabado de la piedra, y recubre zonas con suciedad, fragmentaciones y morteros de juntas. Se encuentra repartido irregular y puntualmente por toda la Cruz y, aunque tiene cierto gro-

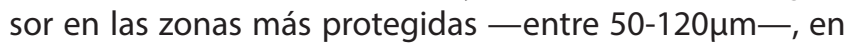
la mayoría de los casos su aspecto actual es de veladura. Por su disposición podría considerarse el más moderno de los dos.

El de tonalidad amarillenta, cuando se conserva, se encuentra normalmente en los volúmenes más internos del capitel [Figura 4B]. Está aplicado tanto por encima del relieve como en pérdidas de volumen, lo que evidencia que no es de origen. Este recubrimiento cromático tiene una textura lisa pero fisurada, su grosor oscila entre las 200$400 \mu \mathrm{m}$, y en su superficie se observan restos de suciedad y costras negras. Análisis microquímicos sobre muestra con ácido clorhídrico apuntan a una composición carbonatada. Podría tratarse de un recubrimiento a la cal teñida con pigmentos tierras y escasa proporción de áridos silíceos muy finos. Aunque actualmente solo se conserva en el capitel también se debió aplicar en la cruz, ya que al analizar el reverso de una costra negra de esta zona se observaron restos puntuales.
Cabe indicar que en un punto de una de las muestra del recubrimiento amarillento, se ha observado un estrato de blanco por debajo, también de composición carbonatada y escasa proporción de áridos silíceos finos, que no se corresponde con otros recubrimientos o morteros aquí observados, por lo que podría tratarse de un encalado anterior [Figura 4C].

\section{Estado de conservación}

\section{Depósitos superficiales, costras y pérdidas de volumen}

El conjunto presentaba un estado de conservación muy variable según la pieza concreta y su orientación actual ${ }^{7}$. Visualmente destacaba la gran diferencia de acumulación de depósitos superficiales entre la zona superior — cruz y capitel—y la inferior — fuste y basa-.

Sobre el material pétreo y de manera general, las principales alteraciones se relacionaban con pérdidas de volumen por pequeñas descamaciones, fragmentaciones, alveolizaciones y diferentes depósitos superficiales de suciedad compactada, recubrimientos biogénicos $y$, puntualmente, costra negra dendrítica.

La cruz presentaba en casi toda su superficie pequeñas descamaciones con pérdida de volumen y, en la cara noroeste, costras de suciedad compactada, recubrimientos biogénicos y costra negra dendrítica -localizada exclusivamente en uno de los calados de los brazos horizontales- [Figuras 5A, B, C, D, H].

En el capitel, además de alteraciones similares a las de la cruz, la diferencia entre la orientación noroeste y suroeste era mayor. La cara suroeste presentaba alveolización y arenización localizadas, sobre todo en una de las figuras de los Santos [Figura 5E]. Además, se podía distinguir fácilmente la presencia de costra negra en las zonas más internas de relieve, donde no se había producido lavado del agua de lluvia y en donde se conservaba el recubrimiento cromático de tonalidad amarillenta. En estos casos su textura era dendrítica, tenía gran adherencia con el sustrato y su grosor oscilaba entre $200-400 \mu m$. La composición car-
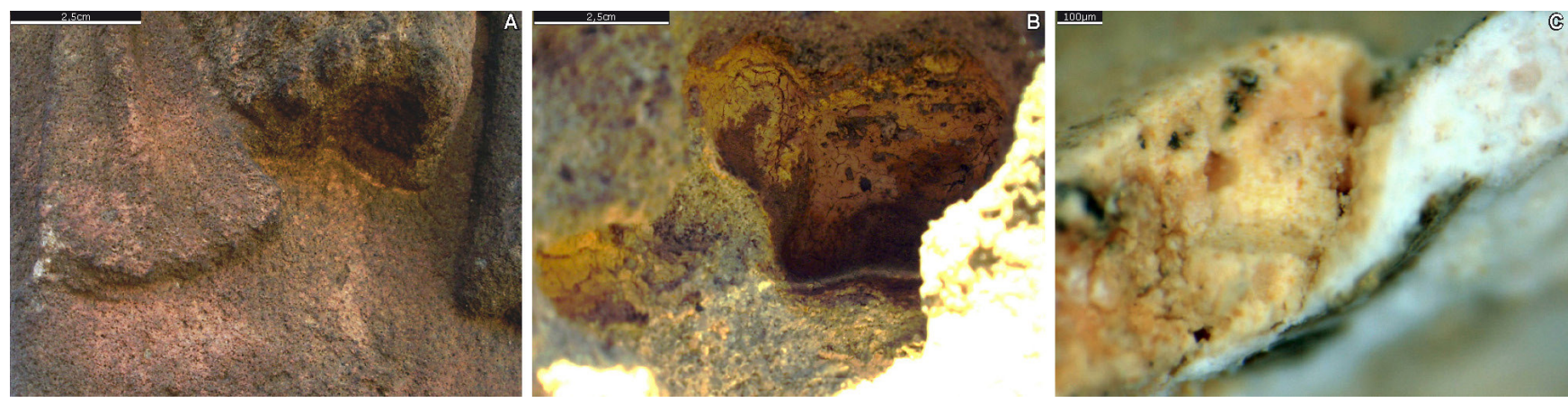

Figura 4. Recubrimientos cromáticos artificiales: (A) macrofotografía de cromatización rosa, (B) macrofotografía de cromatización ocre, (C) imagen a microscopio estereoscópico de sección de cromatización ocre. @ Manuel A. Iglesias Campos. 

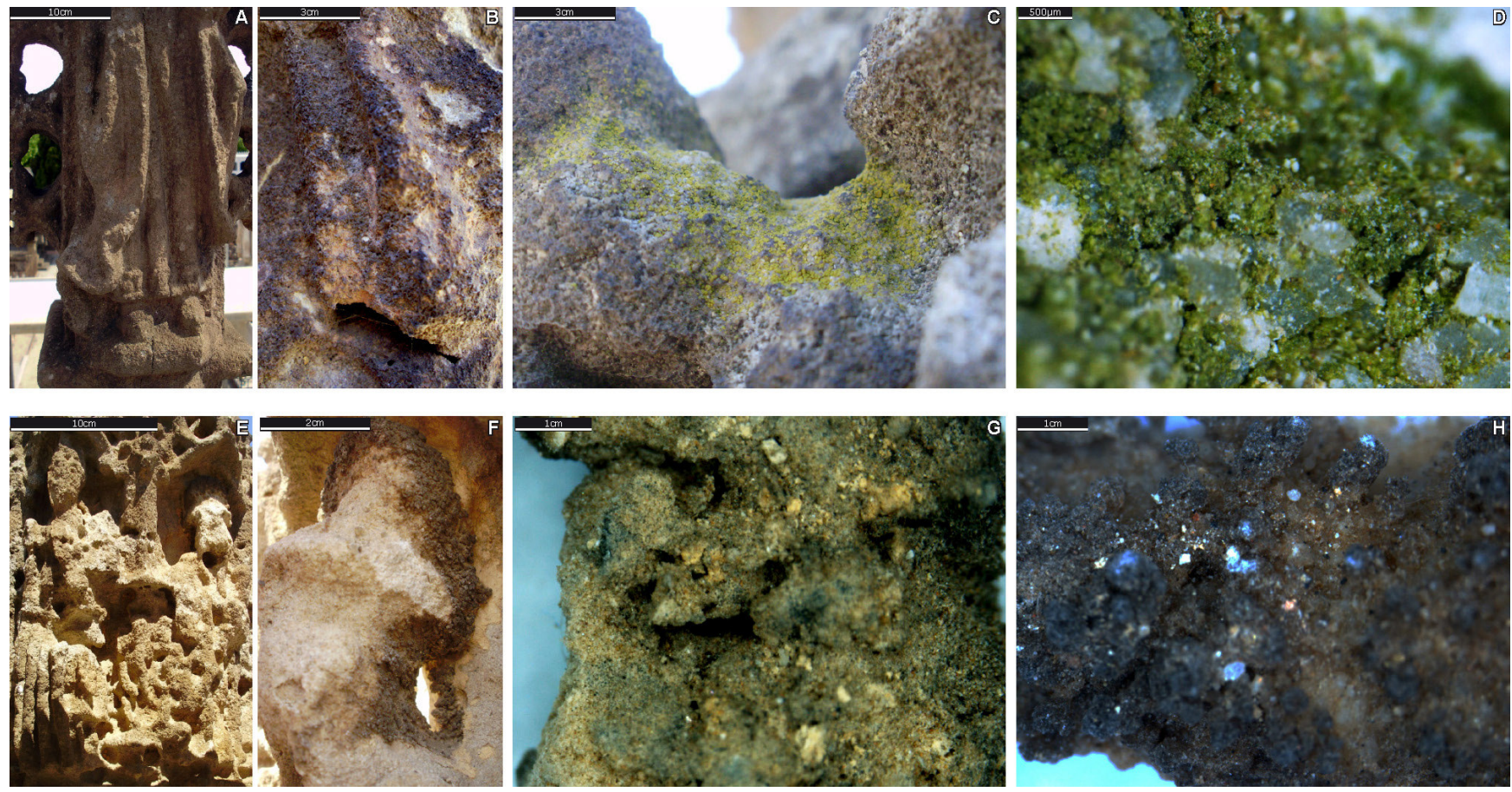

Figura 5. Alteraciones cruz y capitel: (A) fotografía de depósitos superficiales y descamaciones, (B) detalle de depósitos superficiales y descamaciones, (C) macrofotografía de crecimientos liquénicos, (D) imagen a microscopio estereoscópico de crecimientos liquénicos, (E) fotografía de alveolizaciones y descohesión del material pétreo, (F) macrofotografía de costra negra dendrítica y descohesión del material pétreo, $(\mathrm{G})$ imagen a microscopio estereoscópico del reverso de costra negra dendrítica, $(\mathrm{H})$ imagen a microscopio estereoscó $\neg$ pico del anverso de costra negra dendrítica. ๑ Manuel A. Iglesias Campos.

bonatada del recubrimiento cromático amarillento había favorecido este proceso de sulfatación intenso [Figura 5F]. Es necesario remarcar la relación entre la costra dendrítica y este recubrimiento cromático ya que, al observar con microscopio estereoscópico una muestra de costra negra del interior de uno de los brazos de la cruz, donde no se conservaba este recubrimiento, en el reverso sí que se pudieron observar restos puntuales [ Figura 5G].

El fuste tenía un mejor estado de conservación, con pequeñas pérdidas de materia por disyunción, abrasión o golpes, y una capa de suciedad muy superficial; en cambio, la basa presentaba cierta erosión diferencial y estaba limpia. Por comparación, la diferencia de suciedad con las partes superiores parecía indicar que el fuste había tenido un tratamiento de limpieza mayor, quizás en el último traslado y montaje, y que la basa, aparte de una posible limpieza como la del fuste, se limpiaba habitualmente durante el riego del parterre en el que se ubica el conjunto.

Todos los morteros, en cambio, tenían un buen estado de conservación y no se apreciaban degradaciones significativas. Solo puntualmente en los conservados en la cruz y en el capitel aparecían costras de suciedad compactada y costra negra dendrítica, pero ninguno presentaba problemas significativos de disgregación. Es necesario destacar la diferencia de desgaste entre el mortero de unión de la cruz con el capitel y la piedra del capitel porque indicaba que el material pétreo se había ido degradando más que el mortero después de su colocación.

\section{Biodeterioro}

La presencia más importante de indicadores de biodeterioro se localizaba en la cruz y en el capitel [Figuras 5C, D]. De manera generalizada se observaban organismos casmoendolíticos, principalmente hongos y líquenes, que habían colonizado la textura microrugosa de la piedra y que parecían haber provocado pequeñas microfisuras, posiblemente por la alternancia de periodos de hidratacióndeshidratación. En el caso de las formaciones liquénicas, la mayoría masas granulares y pulverulentas, el grado de adherencia era muy diferente. En la cara norte de la cruz también se documentaron recubrimientos más o menos uniformes de algas epilíticas y endolíticas, coincidiendo con zonas puntuales de descamación. La presencia de briofitos, y más en concreto de pequeñas colonias de musgos, se restringía a las partes más internas y profundas del relieve escultórico, sobre todo en la cara norte del capitel, ya que habían aprovechado una ligera acumulación de depósitos terrosos superficiales para establecerse en esas zonas.

Estos indicadores de biodeterioro, además de cambios cromáticos dependiendo del periodo estacional, parecían haber favorecido una cierta descohesión del material pétreo por la acción de sus elementos de fijación y por los subproductos derivados de su metabolismo.

Además del biodeterioro que afectaba directamente a la Cruz, hay que mencionar el del basamento de mamposte- 
ría. En él suelen crecer diferentes especies de plantas superiores, algunas trepadoras, aprovechando las pérdidas de mortero entre sus piedras. Aunque se recortan o eliminan de manera periódica durante los trabajos de mantenimiento del recinto, vuelven a desarrollarse rápidamente al aprovechar las condiciones apropiadas que el espacio les ofrece. Respecto a este tipo de biodeterioro, la basa era la parte más afectada y, aunque en el momento de realizar el estudio no había plantas superiores, se pudieron observar restos de raíces aéreas de especies trepadoras adheridas a su superficie.

También, aunque de manera muy puntual y casi exclusivamente en la cruz y en el capitel, existían depósitos de guano, con una elevada adherencia al sustrato, y nidos de arácnidos.

\section{Sales solubles}

Como algunas de las alteraciones parecían estar relacionadas con la presencia de sales solubles, se realizó una campaña de apósitos con fibras de celulosa y agua desionizada en las cuatro caras de la Cruz. Los puntos se seleccionaron según las formas de alteración predominantes que pudiesen relacionarse con presencia de sales - principalmente alveolizaciones y descamaciones en este caso- - Es necesario indicar que en el momento de colocar los apósitos casi no se observó absorción de agua en la piedra. Esta escasa penetración, como se ha comentado, se pudo comprobar en laboratorio sobre alguna de las muestras mediante análisis por absorción de gota de agua que indicaban la existencia de un producto que reducía su penetración -y que deberá tenerse en cuenta al evaluar los resultados de los apósitos-.

Los valores diferenciales de conductividad no mostraron presencia significativa de sales solubles. Las medidas eran muy bajas - promedio de $8,71 \mu \mathrm{s}$ - y parecían indicar que las sales solubles no estaban relacionadas, a priori, con los procesos de descamación puntual ni con las alveolizaciones del capitel. Con estos resultados, el origen de las descamaciones de la cruz y del capitel parecía relacionarse más con procesos de biodeterioro, y principalmente de los derivados de organismos epilíticos y endolíticos ${ }^{8}$. En cambio, la alveolización de la cara suroeste del capitel podría estar más vinculada a procesos de erosión eólica con orientación hacia la zona afectada.

Al obtener estos valores tan bajos de conductividad se decidió medir el pH de la solución. Los resultados obtenidos — promedio de 5,63 - suelen ser relativamente habituales en areniscas silíceas y no permiten relacionar su origen con residuos de contaminación o efectos de lluvia ácida.

De todos modos, como los resultados eran algo extraños y para relacionarlos con los valores obtenidos en los apósitos, ya en laboratorio, aprovechando las pequeñas muestras de piedra, se pusieron dos de los fragmentos en agua desionizada durante 24 horas para medir conductividad y $\mathrm{pH}$. Los resultados indicaban que cerca de las zonas alveolizadas de la cara suroeste del capitel había una presencia moderada de sales solubles - $290 \mu s$ - pero que, debido al posible recubrimiento observado en las muestras, el agua del apósito no había penetrado y, por tanto, no las había absorbido. Los valores de $\mathrm{pH}$ de las soluciones dieron valores más bajos que los de los apósitos, en torno a $4,88^{9}$.

Esta información se tuvo en cuenta para plantear la intervención y los controles posteriores, y se decidió realizar nuevas mediciones tras la limpieza para establecer comparativas - los resultados se exponen en el apartado de tratamiento-.

\section{Hipótesis}

Como se ha comentado, no se conserva ninguna documentación sobre intervenciones anteriores, pero el estudio de los materiales y de las alteraciones, y las fechas de los traslados, permiten establecer determinadas hipótesis sin que, evidentemente, supongan afirmaciones sobre la historia del monumento.

Así, hacia principios del siglo XIX, tras haber estado ubicada desde su construcción en el límite entre Teià y AleIla, la Cruz se traslada a la necrópolis de la iglesia de Sant Pere. La ubicación de los dos morteros de cal hace pensar que puedan ser de este momento [Figura 6]. A su vez, el recubrimiento cromático de tonalidad amarillenta, que aparece tanto por encima de la piedra y de los morteros de cal como en el interior de las alveolizaciones y pérdidas de volumen, también podría corresponder a este traslado. Pensar en una degradación progresiva de la Cruz desde su construcción -año 1500 según inscripción- y una posible adecuación al colocarse en el nuevo espacio, no parece una proposición del todo equivocada.

Posteriormente se vuelve a trasladar, sin saber la fecha exacta de su colocación, al incluirla Miquel Garriga i Roca en el diseño de 1868 para el nuevo cementerio. De este momento podrían ser los morteros hidráulicos de coloración rosácea que aparecen encima de los de cal y también lo podría ser el recubrimiento cromático de la misma tonalidad que rellena la textura de acabado de la piedra y recubre zonas con suciedad, fragmentaciones y morteros de junta [Figura 6]. En este momento también podría haberse realizado una limpieza con algún tratamiento agresivo que no llegaría a ser totalmente efectivo - como demuestran los restos de costras negras dendríticas que quedan sobre el recubrimiento cromático amarillento y ciertos depósitos compactados bajo el recubrimiento rosáceo- También se podría haber aplicado un producto protector para reducir los efectos del agua en el material. Pensar en una intervención importante para adecuar la Cruz al nuevo cementerio, teniendo en cuenta que en él se construyeron panteones de gran valor artístico, tampoco parece una idea del todo errónea. 

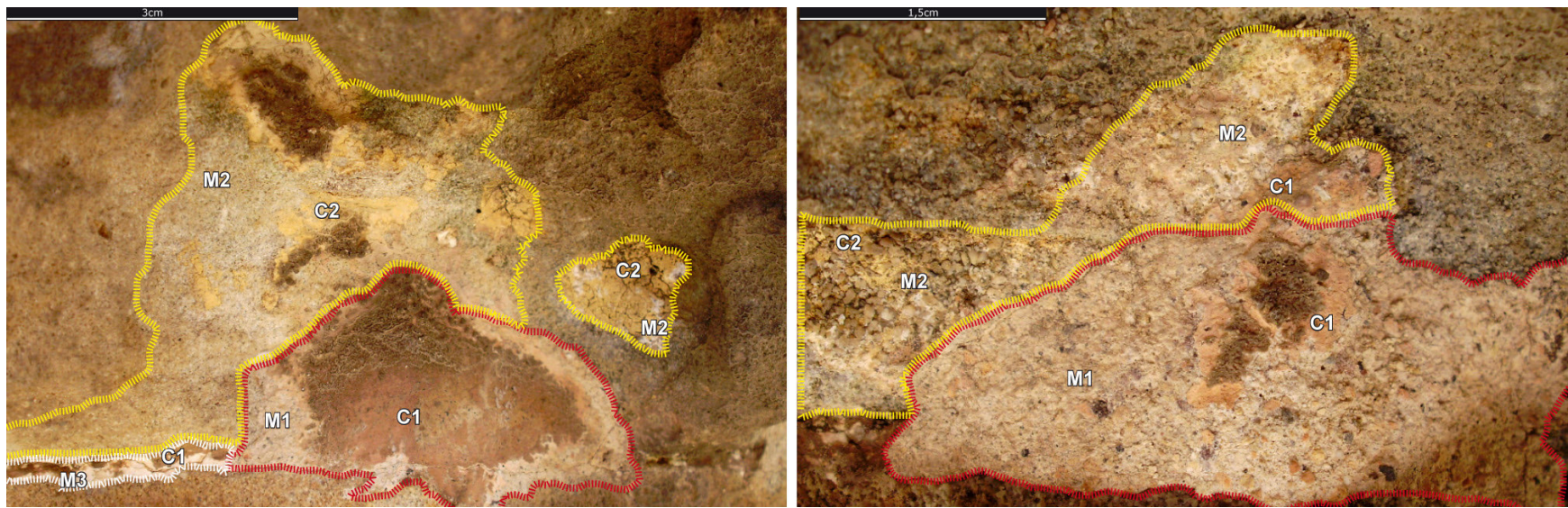

Figura 6. Disposición y estructura de morteros y recubrimientos cromáticos artificiales: (M1) mortero rosáceo, (M2) mortero de cal, (M3) mortero de cal sin apenas áridos, (C1) cromatización rosa, (C2) cromatización ocre. @ Manuel A. Iglesias Campos.

En el siglo XX, aparte de la colocación de tres placas conmemorativas en el basamento, no se tiene referencia de ninguna intervención. En aquel momento y en trabajos posteriores de mantenimiento se pudieron colocar los morteros de cemento que existen entre las juntas del basamento. Es de suponer también que, debido a la diferencia de suciedad entre basa-fuste y capitel-cruz, en riegos de mantenimiento de la zona ajardinada en la que se ubica la Cruz, se ha podido realizar alguna limpieza con manguera y agua.

\section{Intervención}

Los objetivos generales de la intervención se centraron en la limpieza y saneamiento de la Cruz, asegurando la conservación de las intervenciones anteriores -morteros y recubrimientos cromáticos-. Su ubicación en el exterior, en un espacio de necrópolis donde la mayor parte de mausoleos y esculturas tienen problemas similares, impide plantear una solución efectiva sin pensar, además, en un seguimiento y en un mantenimiento.

Debido a las características de los materiales y alteraciones se priorizó la limpieza mecánica en seco. Primero se eliminaron mediante pincel, cepillo y aspirador los depósitos menos coherentes y, posteriormente, se emplearon bisturí y micromotor - de manera puntual-y microproyección de abrasivos a baja presión - general- para retirar los depósitos de suciedad compactados, las costras negras y los recubrimientos biogénicos. Antes de la primera limpieza se preconsolidaron dos áreas muy descohesionadas del capitel mediante silicato de etilo con biocida - Bio Estel CTS ${ }^{\circledR}$ - El producto se aplicó a pincel y la zona se selló durante 15 días con film de polietileno para evitar una rápida evaporación.

En la limpieza general se utilizó microabrasímetro con deshumidificador de aire y compresor de $3 \mathrm{CV}-\mathrm{y}$ boquilla recta de $1,2 \mathrm{~mm}$ de diámetro. Además de la suciedad compactada y las costras negras, se intenta- ba retirar -o afectar- el recubrimiento cristalino no pétreo porque reducía la transpiración del material. Se necesitaba, por tanto, un mecanismo de limpieza en el que predominase una erosión controlable. Esto se tuvo especialmente en cuenta para la selección final del producto y del ángulo de la microproyección.

Con relación al producto, debido al tamaño de la boquilla que limitaba la granulometría de los abrasivos a utilizar y a las características de la suciedad, se hicieron diversas pruebas con silicato de aluminio $80-160 \mu \mathrm{m}$ y piedra pómez $188 \mu \mathrm{m}$ a diferentes presiones, ángulos y distancias. Se descartaron otros abrasivos habituales como microesfera de vidrio, vidrio micronizado u óxido de aluminio por ineficacia frente al tipo de recubrimiento a eliminar, por tamaño o por dureza, respectivamente. Finalmente se utilizó silicato de aluminio para trabajar con una partícula aristada y laminar, en comparación con la morfología más globular de la piedra pómez, porque favorecía el efecto buscado. La piedra pómez se descartó también porque, debido a su elevada friabilidad, generaba una gran cantidad de polvo en el área de trabajo que se adhería a la superficie, y aunque se podía retirar con relativa facilidad mediante aspiración, posibles residuos que pudieran quedar en intersticios y fisuras resultarían, por su higroscopicidad, perjudiciales para los materiales (Iglesias et al. 2006, p. 686). Con relación al ángulo, a pesar de que estudios recientes indican que el ángulo que menos modificaciones provoca en materiales detríticos es el de $75^{\circ}$ y el que más el de $45^{\circ}$ (Iglesias-Campos et al. 2014, IglesiasCampos 2014), se prefirió emplear esta última opción ya que se pretendía afectar, y en lo posible retirar, el tratamiento superficial que impermeabilizaba la superficie.

Evidentemente, el estado de conservación determinó la presión -entre 0,5-2bar-y la distancia -entre $5-20 \mathrm{~cm}-$, que se fueron modificando según el grado de alteración del material y la dureza de los depósitos a retirar [Figura 7]. 

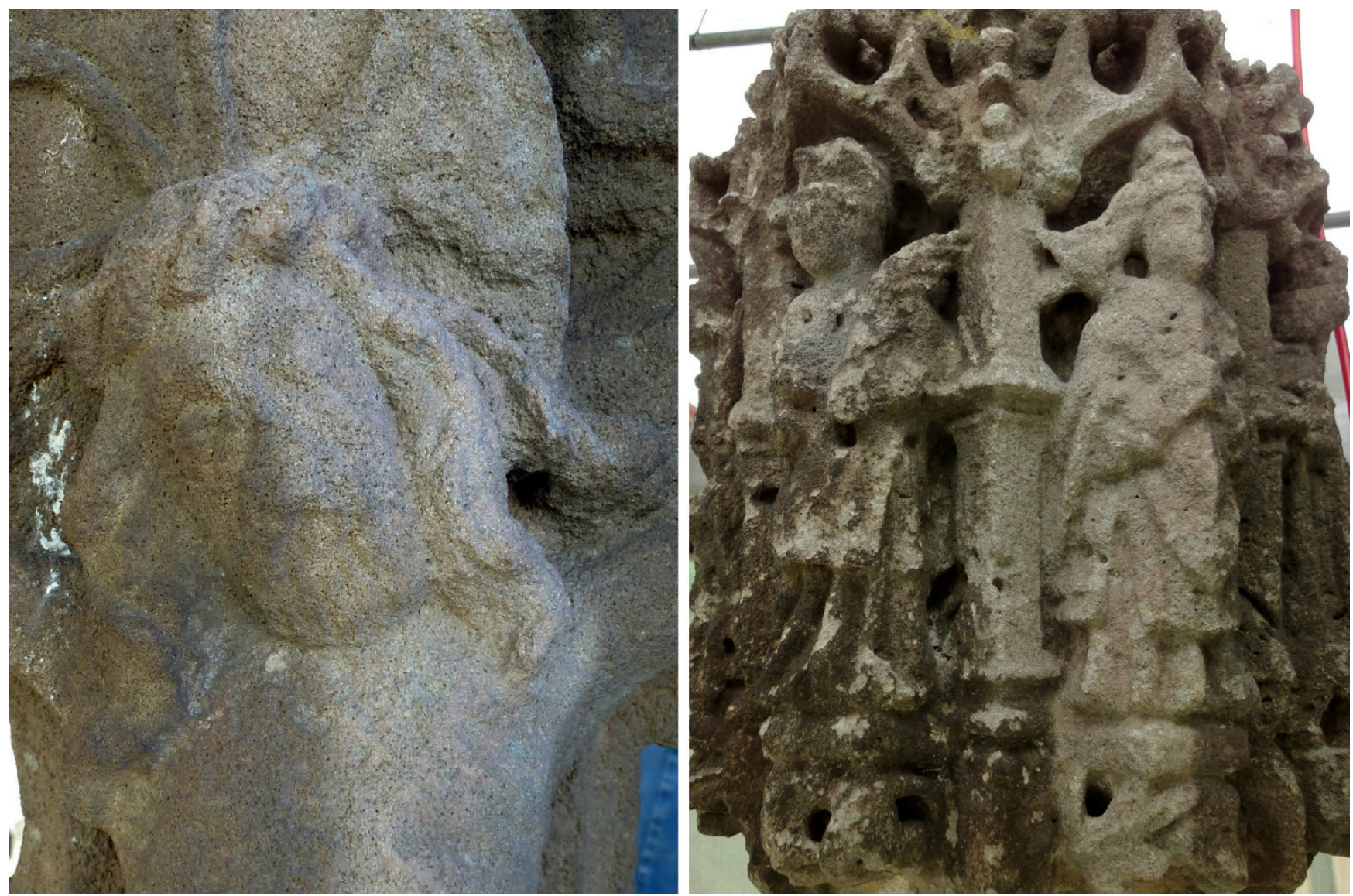

Figura 7. Limpieza con microproyección: detalle de la cruz (izquierda) y del capitel (derecha). @ M. Núria Avecilla Palau.

La limpieza se realizó en sentido descendente. En la cruz, la microproyección se realizó entre 0,5 y 1,5bar ya que no existía disgregación importante del sustrato. En el capitel, donde la descohesión era más acusada, la presión no superó los 0,5bar y, según la zona, se aumentaba ligeramente al reducir la distancia de trabajo - entre $10-20 \mathrm{~cm}$ en las zonas más alteradas y entre $5-10 \mathrm{~cm}$ en el resto-. En el fuste, en mejor estado de conservación, se pudo trabajar con presiones de 1,5-2bar; y en la basa, con erosiones diferenciales, a 1 bar.

Tras la limpieza en seco, y de manera puntual y selectiva, se realizó una limpieza química —Desogen ${ }^{\circledR}$ al 2\% neutralizado posteriormente con agua desionizadaen la cara norte de la cruz y capitel donde la microproyección de abrasivos no eliminaba totalmente los recubrimientos biogénicos.

Finalizada la limpieza, tal y como se ha comentado al hablar de las sales solubles, se realizó una nueva campaña de apósitos en los mismos puntos que en el estudio preliminar. A diferencia de la primera campaña, al colocarlos se observaba visualmente una ligera absorción de agua en la piedra.

Los valores diferenciales de conductividad continuaron siendo bajos — promedio de $47 \mu \mathrm{s}$ - , aunque más elevados que en la primera campaña. Resulta cuando menos curioso que después de limpiar la suciedad superficial, en la que están depositadas sales ambientales, aumenten ligeramente las medidas de conductividad. Estos resultados parecen indicar que con la limpieza mecánica se había eliminado parcialmente el producto que reducía la penetración de agua, favoreciendo un intercambio interior-exterior en el material. Los valores de $\mathrm{pH}$ fueron ligeramente más bajos, aunque sin modificaciones sustanciales — promedio de 5,45-. Al igual que en la primera campaña, se puso un fragmento de piedra de la zona alveolizada en agua desionizada que continuaba indicando una presencia moderada de sales, pero más elevada que en los apósitos - 157 $\mu \mathrm{s}-$, y un $\mathrm{pH}$ similar, aunque ligeramente más bajo $-5,22$ -

La reintegración se limitó a las oquedades provocadas por alteración, buscando un sellado que redujera la acumulación de agua, la de depósitos de partículas ambientales o la formación nidos de insectos. En algunas partes del capitel se reintegraron también líneas decorativas deterioradas que modificaban el diseño escultórico y, por tanto, la caída de agua [Figura 8].

Las descamaciones y pequeñas fisuras se consolidaron internamente con inyecciones de mortero fluido - PLM$\mathrm{S}^{\oplus}$ - Este mortero se empleó también en el sellado de las fisuras, en el biselado de las descamaciones, y en las pequeñas reintegraciones de volumen, adicionándole 

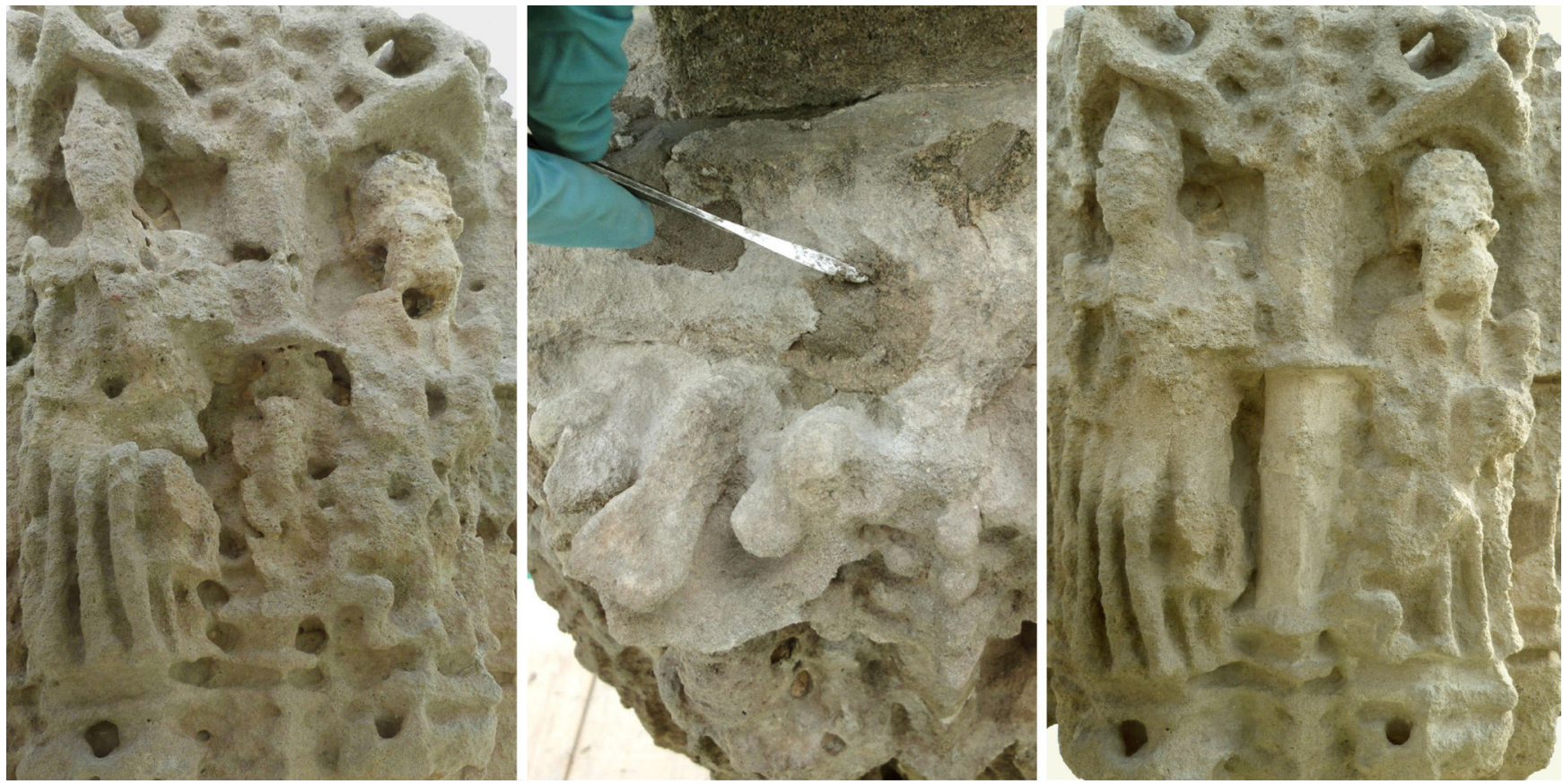

Figura 8. Reintegración: capitel después de la limpieza (izquierda), reintegración de descamaciones y pequeñas pérdidas de volumen (centro) y capitel después de la reintegración. @ M. Núria Avecilla Palau.

áridos silíceos y carbonatados, de diferente color y de tamaño arena fina, en una proporción aproximada de 1:3. Según la zona a reintegrar, el mortero se ajustaba de color variando el porcentaje de los áridos.

Finalmente y de manera puntual, se realizó una consolidación en las áreas más alteradas del capitel, fuste y basa, mediante silicato de etilo con biocida - Estel $1200^{\circledR}$ - aplicado con pincel, dejándolo actuar durante 15 días y sellando la zona con film de polietileno.

\section{Conclusiones}

Adaptando algunas de las propuestas sobre el tema (Wedekind et al. 2011) a las necesidades de la obra, y después de seis meses desde la finalización de la intervención, se ha iniciado el programa de conservación preventiva y mantenimiento que ha de permitir estudiar la evolución de la Cruz a partir del registro de las alteraciones, de los tratamientos realizados y de los depósitos de suciedad que se vayan formando.

Se ha realizado un primer control, a modo de prueba, para definir una propuesta según las exigencias reales de la obra; porque este programa, para ser viable y efectivo, deberá adaptarse a los medios técnicos y económicos disponibles. El primer seguimiento se centró en tres aspectos: 1) controlar la evolución de las alteraciones documentadas y registradas en los estudios previos; 2 ) valorar el estado de los morteros aplicados como medida preventiva para evitar la acumulación del agua y la acción erosiva del viento; 3 ) revisar la presencia de los excrementos de aves, nidos de insectos y crecimiento de plantas superiores.

Se ha realizado además una limpieza manual mediante pincelado de los depósitos superficiales -evidentemente sin adherencia a la superficie-y con bisturí y cepillado con agua desionizada - de depósitos puntuales de guano-, y una nueva campaña de apósitos - con resultados similares a los de la segunda: conductividad promedio de 56,5 $\mu \mathrm{s}$, y pH 5,54-. Toda esta información se ha recogido en tablas y fotografías comparativas de patologías y tratamiento.

A partir de los resultados - positivos ciertamente por el escaso tiempo transcurrido desde la intervención-, se prevé realizar controles anuales -o más espaciados en el tiempo dependiendo de la evolución-que tendrían una duración máxima de tres-cuatro días. El coste será asumido por el Programa de Conservación y Restauración de la Oficina de Patrimonio Cultural de la Diputación de Barcelona - personal técnico, material y posibles análisis-y por el Ayuntamiento de El Masnou -infraestructura, andamiaje y eliminación de plantas superiores del basamento-. Anualmente, tras cada control, se emitirá un informe detallado de los resultados.

Desde la propuesta que aquí se ha planteado, y en momentos en los que las instituciones responsables del patrimonio inmueble tienen que racionalizar los recursos disponibles, plantear estudios acordes con las necesidades reales de la intervención, que faciliten información suficiente para llevar un seguimiento y un mantenimiento posterior, permite actuar según los criterios establecidos en este ámbito. 


\section{Notas}

[1] El cementerio cuenta con panteones de gran valor artístico en los que participaron importantes arquitectos catalanes de los siglos XIX y XX, como Bonaventura Bassegoda, Pere Jordi Bassegoda o Josep Llinàs, y escultores como Josep Llimona, Frederic Marés, Rafael Atché o Josep Maria Subirachs, entre otros.

[2] Según Decreto 14/3/1963 número 571/63: decreto de protección de los escudos, emblemas, piedras heráldicas, rollos de justicia, cruces de término y piezas similares de interés HistóricoArtístico.

[3] Por su grosor, se ha optado por emplear el término recubrimiento cromático artificial para definir las capas externas dadas por el hombre que aparecen en este monumento, aunque el concepto aparece definido en algunos glosarios como pátina artificial (AA.VV 2013, ICOMOS-ISCS 2008).

[4] Durante la exposición se utilizará el término Cruz —en mayúsculas - para referirse a la Cruz de Término como conjunto, y cruz —en minúsculas - para hablar de la cruz de coronamiento.

[5] No existe documentación de cómo sería el basamento original ni de su altura aunque es de suponer que, según los modelos habituales, podrían haber sido unas gradas de planta circular o poligonal.

[6] No existe documentación de cómo se realizaron los dos traslados de la Cruz aunque es de suponer una separación de las diferentes partes, tal y como parece confirmar el estudio de los morteros y de los sistemas de unión.

[7] Es necesario recordar que, tal y como se ha comentado, se desconoce si la disposición y orientación actuales se corresponden con las iniciales.

[8] Como en muchas alteraciones, siempre es difícil establecer un único o primer origen; pero, aunque las pequeñas descamaciones en este material pétreo puede relacionarse con otros agentes y mecanismos intrínsecos - como una presencia mayor de componentes arcillosos o carbonatados-, los crecimientos algales epilíticos y endolíticos detectados en las descamaciones de la cruz y del capitel parecían apuntar a un deterioro favorecido por estos organismos (Ascaso, Wierzchos y Castello 1998).

[9] Los valores de $\mathrm{pH}$ obtenidos por inmersión de fragmentos de piedra en agua desionizada, mucho más bajos que en los apósitos, junto con la descohesión de las fases de unión y el relativo deterioro de los granos observados en el reverso de las muestras, podrían hacer pensar en una limpieza no documentada con productos agresivos.

\section{Bibliografía}

ACTIUM PATRIMONI CULTURAL, SL (2010). Memòria mapa del patrimoni cultural i natural. El Masnou, El Maresme. Area de Cultura. Diputació de Barcelona. <http://patrimonicultural.diba.
cat/uploads/08118/memoria_integra/_Mem\%C3\%B2ria\%20in tegra_0611139354eec4cb2c22744725fb1b4d.pdf> [consulta 10/07/2014].

ASCASO, C.; WIERZCHOS, J.; CASTELLO, R. (1998) "Study of the biogenic weathering of calcareous litharenite stones caused by lichen and endolithic microorganisms", International Biodeterioration \& Biodegradation, 42 (1): 29-38

AA.VV. (2013). Proyecto Coremans: Criterios de intervención en materiales pétreos. Madrid: Secretaría General Técnica. Centro de Publicaciones. Ministerio de Educación, Cultura y Deporte. $<$ https://sede.educacion.gob.es/publiventa/descargas.action?f_ codigo $=14516 C \&$ codigoOpcion $=1>$. [consulta 10/07/2014].

AA.VV. (2003). “Criterios de intervención en materiales pétreos: conclusiones de las Jornadas celebradas en febrero de 2002 en el IPHE". Bienes Culturales: Revista del Instituto del Patrimonio Histórico Español, 2: 1-34 <http://ipce.mcu.es/pdfs/CriteriosMaterialesPetreos.pdf $>$. [consulta 10/07/2014].

BASTARDES, A. (1983). Les Creus al vent. Col•lecció Aire Lliure 1. Barcelona: Editorial Milla.

ASHURST, N. (1994). Cleaning Historic Buildings. Vol. 1. Substrates, Soiling and Investigation. London: Donhead Publishing.

ESBERT, R. M. ; ORDAZ, J. ; ALONSO, F. J. (1999) Anàlisi i pautes d'actuacions en els elements de pedra de les façanes de l'Eixample de Barcelona. Barcelona: Pro-Eixample.

FARRANDO BOIX, R. (1999). La pedra de Montjuïc de la Catedral de Barcelona. Barcelona: Publicació La Busca.

GÓMEZ-GRAS, D.; PARCERISA, D.; CALVET, F., PORTA J.; SOLÉ DE PORTA N.; CIVÍS, J. (2001) "Statigraphy and petrology of the Miocene Montjuïc delta (Barcelona, Spain)", Acta Geológica Hispánica, 36, 1-2: 115-136. <http://revistes.ub.edu/index.php/ActaGeologica/article/viewFile/4787/6255>. [consulta 15/07/2014].

ICOMOS-ISCS (2008). Illustrated glossary on stone deterioration patterns. Paris: ICOMOS-International Documentation Centre. <http://www.icomos.org/publications/monuments_and_sites/15/pdf/Monuments_and_Sites_15_ISCS_Glossary_Stone. pdf $>$ [consulta 15/07/2014].

IGLESIAS-CAMPOS, M.A. (2014). "Effects of mechanical cleaning by manual brushing and abrasive blasting on lime render coatings on Architectural Heritage", Materiales de Construcción, 64, 316, e039. <http://materconstrucc.revistas.csic.es/index.php/ materconstrucc/article/view/1672/2019> [consulta 15/12/2014]

IGLESIAS-CAMPOS, M.A.; GARCÍA FORTES. S.; PRADA PÉREZ, J.L. (2014). "Influence of projection angle in sandblasting cleaning on detrictic stone materials in Architectural Heritage", Materiales de Construcción, 64, 314, e021. <http://materconstrucc.revistas.csic. es/index.php/materconstrucc/article/view/1475/1721> [consulta $15 / 12 / 2014]$

IGLESIAS CAMPOS, M.Á.; GUASCH FERRÉ, N.; PRADA PÉREZ, J. L.; 
ALCAYDE PALANCA, M. J. (2014). “Análisis de morteros en patrimonio arquitectónico: investigación pluridisciplinar para su conservación". En Jornadas Internacionales Conmemorativas del 80 aniversario del IETCC, CD-ROM. Madrid: Instituto de Ciencias de la Construcción Eduardo Torroja - Consejo Superior de Investigaciones Científicas.

IGLESIAS, M.; GEA, B.; PRADA, J.L; GUASCH, N. (2006). “Low-pressure abrasive cleaning of historic building materials". En Proceedings of the International Heritage, Weathering an Conservation Conference (HWC-2006), Vol. 2, London: Taylor \& Francis Group, 681-686.
RICO VÁZQUEZ, M.; ROIG LERONE, M. (2008). “El cementiri del Masnou, un museu a l'aire lliure (segles XVIII-XXI). III Beca d'Investigació i de Recerca Local del Masnou, 2006. Roca de Xeix, 27.

WEDEKIND, W.; RUEDRICH, J.; SIEGESMUND, S. (2011). “Conservation inventory systems for monitoring and protection of cemeteries and tomb façades". En Jardins de pierres. Conservation de la pierre dans les parcs, jardins et cimetières. 14es Journées d'étude de Ia SFIIC. Paris, Institut National du Patrimoine, 22-24 juin 2011. Paris: SFIIC, 181-191.

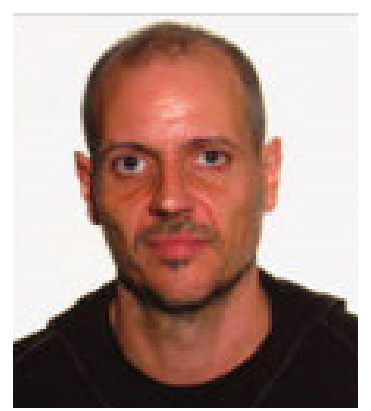

\title{
Manuel Ángel Iglesias Campos
}

Facultat de Belles Arts (Universitat de Barcelona)

manuel.iglesias@ub.edu

Doctor en Conservación-Restauración por la Universidad de Barcelona. Actualmente es profesor de la Secció de Conservació-Restauració de la Facultat de Belles Arts (Universitat de Barcelona) y colabora como docente en diferentes Másteres, Postgrados y cursos profesionales sobre conservación del Patrimonio Histórico. Su línea de investigación se centra en el tratamiento de materiales del Patrimonio Arquitectónico y Monumental.

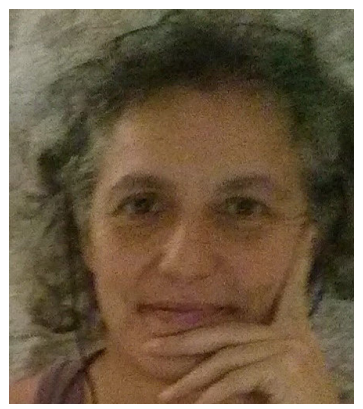

\author{
Núria Avecilla Palau \\ Diputación de Barcelona \\ avecillapn@diba.cat
}

Licenciada en Conservación-Restauración por la Universidad de Barcelona. Profesora de Restauración Arqueológica en la Escuela de Artes y Oficios de la Diputación de Barcelona (1988-2010). Imparte clases de Técnicas Artísticas en el Grado de Historia del Arte de la Universidad Autónoma de Barcelona. Técnica de la Oficina de Patrimonio Cultural de la Diputación de Barcelona. Conservadora Restauradora de Vidrio, Mosaico, Piedra y Pintura Mural Romana. 


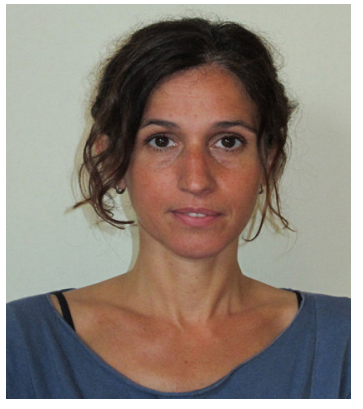

\section{Marta Llach Berné}

Diputación de Barcelona

Ilachbm@diba.cat

Licenciada en Historia del Arte por la Universidad de Barcelona. Diplomada en Restauración de Cerámica y Materiales Pétreos por I'Istituto per l'Arte e il Restauro Palazzo Spinelli de Florencia. Postgrado en Gestión de Empresas Culturales y en Gestión de Proyectos Culturales por la UOC (Universitat Oberta de Catalunya). Técnica de la Oficina de Patrimonio Cultural de la Diputación de Barcelona.

Artículo enviado el 01/08/2014

Artículo aceptado el 14/04/2015 